\title{
SUPPLEMENTARY INFORMATION: A LIKELIHOOD-BASED SCORING METHOD FOR PEPTIDE IDENTIFICATION USING MASS SPECTROMETRY
}

\author{
By Qunhua LI* Jimmy K. EnG ${ }^{\dagger}$ And Matthew Stephens ${ }^{\ddagger}$ \\ Penn State University*, University of Washington ${ }^{\dagger}$ and University of \\ Chicago
}

SI A: Preprocessing procedure. The preprocessing steps are described in detail in Table S1. In brief, the procedure involves clustering together neighboring peaks, by first identifying modes in a histogram formed from the peaks, and then replacing each mode by the highest near-by peak. This reduced set of peaks form the cleaned spectrum. Note that unlike commonly-used thresholding procedures, this procedure is designed not to remove low-intensity peaks, only to pool near-by peaks into a single representative peak. Peak intensities in each cleaned spectrum are then normalized by dividing by the 90th percentile of the intensities of the peaks on the spectrum, to put the peaks from different spectra on a comparable scale. Normalization with respect to significant peaks on the same spectra is commonly used in scoring algorithms; for example, SEQUEST normalizes the top peaks with respect to the highest peak in the whole spectrum [1]. We choose to normalize to the 90th percentile, rather than the highest peak, to reduce sensitivity to outliers. Finally, the normalized intensities are transformed by raising to $1 / 4$ power to stabilize the highly variable intensities.

Clearly, these preprocessing steps involve ad hoc decisions. We therefore experimented with several parameter choices, for example, different combinations of parameters in the cleaning procedure $(b \in\{0.5,1,2\}$ and $c \in\{0.5,1,2\}$ in Table S1), and different transformations (e.g. square root transformation). We found that identification accuracy was not especially sensitive to these choices; the parameter values reported in Table S1 generate the highest identification accuracy on the training spectra. Optimal parameter choices may depend on the type of instrument used to generate the data.

Table S1 shows the preprocessing procedure.

\section{REFERENCES}

[1] Eng JK, McCormack A, Yates JI (1994) An approach to correlate tandem mass spectral data of peptides with amino acid sequences in a protein database. J. Am. 
TABLE S1

Preprocessing procedure.

Let $x_{i}$ and $y_{i}$ denote the location and intensity of peak $i$. For this table only, we order the peaks by their $\mathrm{m} / \mathrm{z}$ values, so $x_{1}<x_{2}<\ldots<x_{n}$.

1. Bin the peaks on a spectrum according to peak locations with prespecified binwidth $b$ (e.g. $b=2 D a)$. Let $x_{i}^{b}$ be the boundaries of the bins, then $x_{i}^{b}=x_{1}+(i-1) b$, $i=1, \ldots, n^{b}+1$, where $n^{b}=\left\lceil\frac{x_{n}-x_{1}}{b}\right\rceil$ is the number of bins, and $x_{1}$ and $x_{n}$ are the smallest and largest $\mathrm{m} / \mathrm{z}$ values of peaks, respectively.

2. Sum peak intensities in each bin $\left(y_{i}^{b}=\sum_{\left\{j: x_{j} \in\left[x_{i}^{b}, x_{i+1}^{b}\right)\right\}} y_{j}, i=1, \ldots, n^{b}\right)$.

3. Find the bins that are local modes of the sum of peak intensities, defined as $M=$ $\left\{i: y_{i-1}^{b} \leq y_{i}^{b}\right.$ and $\left.y_{i}^{b} \geq y_{i+1}^{b}, i=1, \ldots, n^{b}\right\}$, where $y_{0}^{b}=0$ and $y_{n^{b}+1}^{b}=0$.

4. Find the highest peak within distance $c$ from the center of the bins that are local modes, i.e. $K_{0}=\bigcup_{i \in M}\left\{j: \operatorname{argmax}{ }_{j \in\left[\frac{x_{i}^{b}+x_{i+1}^{b}}{2}-c, \frac{x_{i}^{b}+x_{i+1}^{b}}{2}+c\right)} y_{j}\right\}$ (e.g. $\left.c=2 D a\right)$.

5. Keep peaks within detection range, i.e. $K=\left\{k: 200<x_{k}<2000, k \in K_{0}\right\}$.

6. Normalize peak intensities by $y_{k}^{\prime}=\frac{y_{k}}{y_{0.9}}$, where $y_{0.9}$ is the $90 \%$ percentile of $y_{k}, k \in$ $K$.

7. Transform intensities by $y_{k}^{*}=\left(y_{k}^{\prime}\right)^{\frac{1}{4}}$.

8. Keep $\left(x_{k}, y_{k}^{*}\right), k \in K$ to form the processed spectrum.

Soc. Mass Spectrom 5:976-989.

Department of Statistics

Penn State University

326 Thomas Building

UNIVERSITY PARK, PA 16802, USA

E-MAIL: qunhua.li@psu.edu
Department of Genome Sciences UNIVERSITY OF WASHINGTON Box 355065

SeAtTle, WA 98195, USA

E-MAIL: engj@uw.edu

Department of Statistics and Human Genetics

UNIVERSiTy OF CHICAGO

ECKHART HALL ROOM 126

5734 S. University Avenue

Chicago, IL 60637, USA

E-MAIL: mstephens@uchicago.edu 